\title{
Decomposition of Measure for Marginal Homogeneity in Square Contingency Tables with Ordered Categories
}

\author{
Kouji Yamamoto and Sadao Tomizawa \\ Tokyo University of Science, Japan
}

\begin{abstract}
For the analysis of square contingency tables with ordered categories, Tomizawa et al. (2003) considered a measure to represent the degree of departure from marginal homogeneity (MH). Tomizawa (1993) considered an extended marginal homogeneity (EMH) model. This paper (i) proposes a measure to represent the degree of departure from EMH, (ii) proposes a measure from equality of marginal means (E), and (iii) gives a theorem that the value of measure for $\mathrm{MH}$ is equal to the sum of the value of measure for $\mathrm{EMH}$ and that for E.
\end{abstract}

Zusammenfassung: Für die Analyse von quadratischen Kontingenztafeln mit geordneten Kategorien betrachtete Tomizawa et al. (2003) ein Maß, um den Grad der Abweichung von der marginalen Homogenität $(\mathrm{MH}) \mathrm{zu}$ beschreiben. Tomizawa (1993) betrachtete ein erweitertes marginales Homogeniätsmodel (EMH). Dieser Artikel (i) schlägt ein Maß vor, um den Grad der Abweichung von EMH zu repräsentieren, (ii) schlägt eine Distanz von der Gleichheit von marginalen Mitteln (E) vor, und (iii) liefert einen Satz über die Gleichheit des Wertes von MH und der Summe der Werte von EMH und von E.

Keywords: Extended Marginal Homogeneity, Kullback-Leibler Information, Marginal Mean.

\section{Introduction}

Consider an $r \times r$ square contingency table with the same row and column classifications. Let $p_{i j}$ denote the probability that an observation will fall in the $i$ th row and $j$ th column of the table $(i=1, \ldots, r, j=1, \ldots, r)$.

Consider the marginal homogeneity $(\mathrm{MH})$ model defined by

$$
p_{i \bullet}=p_{\bullet i} \quad \text { for } \quad i=1, \ldots, r,
$$

where

$$
p_{i \bullet}=\sum_{k=1}^{r} p_{i k}, \quad p_{\bullet} i=\sum_{k=1}^{r} p_{k i} ;
$$

see, e.g., Stuart (1955) and Bishop, Fienberg, and Holland (1975, p.282). Let

$$
G_{1(i)}=\sum_{s=1}^{i} \sum_{t=i+1}^{r} p_{s t} \quad \text { and } \quad G_{2(i)}=\sum_{s=i+1}^{r} \sum_{t=1}^{i} p_{s t}
$$

for $i=1, \ldots, r-1$. By considering the difference between the cumulative marginal probabilities, the $\mathrm{MH}$ model may be expressed as

$$
G_{1(i)}=G_{2(i)} \quad \text { for } \quad i=1, \ldots, r-1 .
$$


This states that the cumulative probability that an observation will fall in row category $i$ or below and column category $i+1$ or above is equal to the cumulative probability that the observation falls in column category $i$ or below and row category $i+1$ or above.

Consider the extended marginal homogeneity (EMH) model (Tomizawa, 1993) defined by

$$
G_{1(i)}=\delta G_{2(i)} \quad \text { for } \quad i=1, \ldots, r-1 .
$$

Let $X$ and $Y$ denote the row and column variables, respectively. Consider the model of equality of marginal means (E) defined by

$$
\sum_{i=1}^{r} i p_{i} \bullet=\sum_{i=1}^{r} i p_{\bullet} \quad[\text { i.e., } \mathrm{E}(X)=\mathrm{E}(Y)] .
$$

Tomizawa (1991) pointed out that the MH model holds if and only if both the EMH and the E models hold.

Tomizawa (1995) and Tomizawa and Makii (2001) considered the measures to represent the degree of departure from $\mathrm{MH}$ for the data on a nominal scale, and Tomizawa et al. (2003) considered them for the data on an ordinal scale; see Appendix for the KullbackLeibler (KL) information type measure $\Gamma_{\mathrm{MH}}$ proposed in Tomizawa et al. (2003).

When we want to see the degree of departure from $\mathrm{EMH}$, we cannot use the measure $\Gamma_{\mathrm{MH}}$ because $\Gamma_{\mathrm{MH}}$ can measure the degree of departure from $\mathrm{MH}$, however it cannot measure it from EMH. Therefore, for the data on an ordinal scale, we are interested in a measure to represent what degree the departure from EMH is.

The purpose of this paper is (i) to propose a measure which represents the degree of departure from $\mathrm{EMH}$ (denoted by $\Gamma_{\mathrm{EMH}}$ ), (ii) to propose that from $\mathrm{E}$ (denoted by $\Gamma_{\mathrm{E}}$ ), and (iii) to give the theorem that the value of $\Gamma_{\mathrm{MH}}$ is equal to the sum of the value of $\Gamma_{\mathrm{EMH}}$ and the value of $\Gamma_{\mathrm{E}}$. We emphasize that the measure $\Gamma_{\mathrm{EMH}}$ proposed in this paper is entirely different from the measures which represent the degree of departure from MH in Tomizawa (1995), Tomizawa and Makii (2001), and Tomizawa et al. (2003).

\section{Measures}

\subsection{Measure for Extended Marginal Homogeneity}

We shall consider the measure to represent the degree of departure from EMH. Let

$$
\begin{array}{cc}
\Delta=\sum_{i=1}^{r-1}\left(G_{1(i)}+G_{2(i)}\right) \quad(>0), & G_{1(i)}^{*}=\frac{G_{1(i)}}{\Delta}, \quad G_{2(i)}^{*}=\frac{G_{2(i)}}{\Delta}, \\
\Delta_{U}^{*}=\sum_{i=1}^{r-1} G_{1(i)}^{*}, & \Delta_{L}^{*}=\sum_{i=1}^{r-1} G_{2(i)}^{*} .
\end{array}
$$

Assuming that $\Delta_{U}^{*}>0, \Delta_{L}^{*}>0$, and $\left\{G_{1(i)}+G_{2(i)}>0\right\}$, consider a measure defined by

$$
\Gamma_{\mathrm{EMH}}=\frac{1}{\log 2} I\left(\left\{G_{1(i)}^{*}, G_{2(i)}^{*}\right\} ;\left\{G_{1(i)}^{\mathrm{EMH}}, G_{2(i)}^{\mathrm{EMH}}\right\}\right),
$$


where

$$
\begin{gathered}
I(\cdot ; \cdot)=\sum_{i=1}^{r-1}\left\{G_{1(i)}^{*} \log \left(\frac{G_{1(i)}^{*}}{G_{1(i)}^{\mathrm{EMH}}}\right)+G_{2(i)}^{*} \log \left(\frac{G_{2(i)}^{*}}{G_{2(i)}^{\mathrm{EMH}}}\right)\right\}, \\
G_{1(i)}^{\mathrm{EMH}}=\Delta_{U}^{*}\left(G_{1(i)}^{*}+G_{2(i)}^{*}\right), \quad G_{2(i)}^{\mathrm{EMH}}=\Delta_{L}^{*}\left(G_{1(i)}^{*}+G_{2(i)}^{*}\right) .
\end{gathered}
$$

Note that $I(\cdot ; \cdot)$ is the KL information between $\left\{G_{1(i)}^{*}, G_{2(i)}^{*}\right\}$ and $\left\{G_{1(i)}^{\mathrm{EMH}}, G_{2(i)}^{\mathrm{EMH}}\right\}$.

We see that (i) $0 \leq \Gamma_{\mathrm{EMH}} \leq 1$, (ii) $\Gamma_{\mathrm{EMH}}=0$ if and only if the EMH model holds, and (iii) $\Gamma_{\mathrm{EMH}}=1$ if and only if the degree of departure from EMH is the largest in a sense that $G_{1(i)}=0\left(\right.$ then $\left.G_{2(i)}>0\right)$ or $G_{2(i)}=0\left(\right.$ then $\left.G_{1(i)}>0\right), i=1, \ldots, r-1$ and $\Delta_{U}^{*}=\Delta_{L}^{*}=1 / 2$.

According to the KL information, $\Gamma_{\mathrm{EMH}}$ represents the degree of departure from $\mathrm{EMH}$, and the degree increases as the value of $\Gamma_{\mathrm{EMH}}$ increases.

\subsection{Measure for the Equality of Marginal Means}

We shall consider the measure to represent the degree of departure from the $\mathrm{E}$ model. We note that $\mathrm{E}(X)=\mathrm{E}(Y)$ is equivalent to $\sum_{i=1}^{r-1} G_{1(i)}=\sum_{i=1}^{r-1} G_{2(i)}$ (i.e., $\Delta_{U}^{*}=\Delta_{L}^{*}$ ), although the details are omitted here. Assuming that $\Delta_{U}^{*} \geq 0$ and $\Delta_{L}^{*} \geq 0$, consider a measure defined by

$$
\Gamma_{\mathrm{E}}=\frac{1}{\log 2} I\left(\left\{\Delta_{U}^{*}, \Delta_{L}^{*}\right\} ;\left\{\frac{1}{2}, \frac{1}{2}\right\}\right),
$$

where

$$
I(\cdot ; \cdot)=\Delta_{U}^{*} \log \left(\frac{\Delta_{U}^{*}}{1 / 2}\right)+\Delta_{L}^{*} \log \left(\frac{\Delta_{L}^{*}}{1 / 2}\right)
$$

This may be expressed as

$$
\Gamma_{\mathrm{E}}=1-\frac{1}{\log 2} H\left(\left\{\Delta_{U}^{*}, \Delta_{L}^{*}\right\}\right)
$$

where

$$
H(\cdot)=-\Delta_{U}^{*} \log \Delta_{U}^{*}-\Delta_{L}^{*} \log \Delta_{L}^{*}, \quad[0 \log 0=0] .
$$

Thus, essentially, $\Gamma_{\mathrm{E}}$ represents the Shannon entropy $H\left(\left\{\Delta_{U}^{*}, \Delta_{L}^{*}\right\}\right)$.

We see that $H\left(\left\{\Delta_{U}^{*}, \Delta_{L}^{*}\right\}\right)$ must lie between 0 (when $\Delta_{U}^{*}=0$ then $\Delta_{L}^{*}=1$, or when $\Delta_{L}^{*}=0$ then $\left.\Delta_{U}^{*}=1\right)$ and $\log 2\left(\right.$ when $\left.\Delta_{U}^{*}=\Delta_{L}^{*}=1 / 2\right)$, and therefore $\Gamma_{\mathrm{E}}$ must lie between 0 and 1 . We also see that (i) $\Gamma_{\mathrm{E}}=0$ if and only if there is a structure of $\mathrm{E}$ in the $r \times r$ table, and (ii) $\Gamma_{\mathrm{E}}=1$ if and only if the degree of departure from $\mathrm{E}$ is the largest in a sense that $\Delta_{U}^{*}=0$ (then $\Delta_{L}^{*}=1$ ) or $\Delta_{L}^{*}=0$ (then $\Delta_{U}^{*}=1$ ). Namely, (i) $\Gamma_{\mathrm{E}}=0$ if and only if $\mathrm{E}(X)=\mathrm{E}(Y)$, i.e., $\sum_{i=1}^{r-1} G_{1(i)}=\sum_{i=1}^{r-1} G_{2(i)}$, and (ii) $\Gamma_{\mathrm{E}}=1$ if and only if $\sum \sum_{i<j} p_{i j}=0\left(\sum \sum_{i>j} p_{i j} \neq 0\right)$ or $\sum \sum_{i>j} p_{i j}=0\left(\sum \sum_{i<j} p_{i j} \neq 0\right)$ (i.e., $\operatorname{Pr}(X<Y)=0, \operatorname{Pr}(X>Y) \neq 0$, or $\operatorname{Pr}(X>Y)=0, \operatorname{Pr}(X<Y) \neq 0)$.

According to the KL information or the Shannon entropy, $\Gamma_{\mathrm{E}}$ represents the degree of departure from $\mathrm{E}$ model, and the degree increases as the value of $\Gamma_{\mathrm{E}}$ increases. 


\subsection{Relationships between the Measures}

Assume that $\Delta_{U}^{*}>0, \Delta_{L}^{*}>0$, and $\left\{G_{1(i)}+G_{2(i)}>0\right\}$. Then we obtain the following theorem.

Theorem 1. The value of $\Gamma_{\mathrm{MH}}$ equals the sum of the value of $\Gamma_{\mathrm{EMH}}$ and the value of $\Gamma_{\mathrm{E}}$. Proof. It is easily seen that the right term of equation (1) plus the right term of equation (2) equals the right term of equation (3) in the Appendix. Thus, the proof is completed.

From Theorem 1, $\Gamma_{\mathrm{EMH}}$ is expressed as $\Gamma_{\mathrm{EMH}}=\Gamma_{\mathrm{MH}}-\Gamma_{\mathrm{E}}$. Therefore, the measure $\Gamma_{\mathrm{EMH}}$ also would indicate the degree of departure from $\mathrm{MH}$ excluding the influence of degree of departure from $\mathrm{E}$.

From (1) we see that $\Gamma_{\mathrm{EMH}} \geq 0$. Thus we obtain the next theorem.

Theorem 2. The value of $\Gamma_{\mathrm{MH}}$ is greater than or equal to the value of $\Gamma_{\mathrm{E}}$. The equality holds if and only if there is a structure of EMH in the $r \times r$ table.

From $0 \leq \Gamma_{\mathrm{MH}} \leq 1,0 \leq \Gamma_{\mathrm{E}}<1$ (note that $\Gamma_{\mathrm{E}} \neq 1$ because $\Delta_{U}^{*}>0$ and $\Delta_{L}^{*}>0$ being the assumption), and Theorems 1 and 2, we see that $0 \leq \Gamma_{\mathrm{EMH}} \leq 1$. From $\Gamma_{\mathrm{EMH}}=$ $\Gamma_{\mathrm{MH}}-\Gamma_{\mathrm{E}}$, we see that (i) $\Gamma_{\mathrm{EMH}}=0$ if and only if $\Gamma_{\mathrm{MH}}=\Gamma_{\mathrm{E}}$; namely, the degree of departure from the equality of $G_{1(i)}$ and $G_{2(i)}$ for $i=1, \ldots, r-1$, is equal to the degree of departure from the equality of $\sum_{i=1}^{r-1} G_{1(i)}$ and $\sum_{i=1}^{r-1} G_{2(i)}$. This seems natural when the EMH model holds (i.e., $\Gamma_{\text {EMH }}=0$ ).

We also see that (ii) $\Gamma_{\mathrm{EMH}}=1$ if and only if $\Gamma_{\mathrm{MH}}=1$ and $\Gamma_{\mathrm{E}}=0$; namely, $G_{1(i)}=0$ (then $G_{2(i)}>0$ ) or $G_{2(i)}=0$ (then $G_{1(i)}>0$ ) for $i=1, \ldots, r-1$, and $\mathrm{E}(X)=\mathrm{E}(Y)$. Namely $\Gamma_{\mathrm{EMH}}=1$ indicates that $G_{1(i)} / G_{2(i)}=\infty$ for some $i$ and $G_{1(i)} / G_{2(i)}=0$ for the other $i$, and $\mathrm{E}(X)=\mathrm{E}(Y)$ (i.e., $\sum_{i=1}^{r-1} G_{1(i)}=\sum_{i=1}^{r-1} G_{2(i)}$ ). It seems appropriate to consider that then the degree of departure from EMH is largest.

\section{Approximate Confidence Intervals for the Measures}

Let $n_{i j}$ denote the observed frequency in the $i$ th row and $j$ th column of the $r \times r$ square table $(i=1, \ldots, r, j=1, \ldots, r)$. Assuming that the $\left\{n_{i j}\right\}$ result from a full multinomial sampling, we consider the approximate standard errors and large-sample confidence intervals for $\Gamma_{\mathrm{EMH}}$ and $\Gamma_{\mathrm{E}}$ using the delta method as described by Bishop et al. (1975, Sec.14.6) and Agresti (1990, Sec.12.1). The sample version of $\Gamma_{\mathrm{EMH}}$, i.e., $\hat{\Gamma}_{\mathrm{EMH}}$, is given by $\Gamma_{\text {EMH }}$ with $\left\{p_{i j}\right\}$ replaced by $\left\{\hat{p}_{i j}\right\}$, where $\hat{p}_{i j}=n_{i j} / n$ and $n=\sum \sum n_{i j}$. Similarly, $\hat{\Gamma}_{\mathrm{E}}$ is given. Using the delta method, $\sqrt{n}\left(\hat{\Gamma}_{\mathrm{EMH}}-\Gamma_{\mathrm{EMH}}\right)$ has asymptotically (as $\left.n \rightarrow \infty\right)$ a normal distribution with mean zero and variance,

$$
\operatorname{var}\left[\Gamma_{\mathrm{EMH}}\right]=\frac{1}{\Delta^{2}} \sum_{k=1}^{r-1} \sum_{l=k+1}^{r}\left(p_{k l} w_{k l}^{2}+p_{l k} v_{l k}^{2}\right),
$$

where

$$
\begin{aligned}
& w_{k l}=\frac{1}{\log 2}\left\{\sum_{i=k}^{l-1} \log \left(\frac{G_{1(i)}^{*}}{G_{1(i)}^{\mathrm{EMH}}}\right)-(l-k) \Gamma_{\mathrm{EMH}} \log 2\right\}, \\
& v_{l k}=\frac{1}{\log 2}\left\{\sum_{i=k}^{l-1} \log \left(\frac{G_{2(i)}^{*}}{G_{2(i)}^{\mathrm{EMH}}}\right)-(l-k) \Gamma_{\mathrm{EMH}} \log 2\right\} .
\end{aligned}
$$


Table 1: Occupational status for Japanese father-son pairs

\begin{tabular}{rrrrrrrrrr}
\hline & \multicolumn{10}{c}{ (a) examined in 1955 } \\
Father's status & $(1)$ & $(2)$ & $(3)$ & $(4)$ & $(5)$ & $(6)$ & $(7)$ & $(8)$ & Total \\
\hline$(1)$ & 36 & 4 & 14 & 7 & 8 & 2 & 3 & 8 & 82 \\
$(2)$ & 20 & 20 & 27 & 24 & 11 & 11 & 2 & 11 & 126 \\
$(3)$ & 9 & 6 & 23 & 12 & 9 & 5 & 3 & 16 & 83 \\
$(4)$ & 15 & 14 & 39 & 81 & 17 & 16 & 11 & 15 & 208 \\
$(5)$ & 6 & 7 & 22 & 13 & 72 & 20 & 6 & 13 & 159 \\
$(6)$ & 3 & 2 & 5 & 12 & 18 & 19 & 9 & 7 & 75 \\
$(7)$ & 5 & 3 & 10 & 11 & 21 & 15 & 38 & 25 & 128 \\
$(8)$ & 39 & 30 & 76 & 80 & 69 & 52 & 45 & 614 & 1005 \\
\hline Total & 133 & 86 & 216 & 240 & 225 & 140 & 117 & 709 & 1866 \\
\hline
\end{tabular}

(b) examined in 1975

Son's status

\begin{tabular}{rrrrrrrrrr} 
Father's status & $(1)$ & $(2)$ & $(3)$ & $(4)$ & $(5)$ & $(6)$ & $(7)$ & $(8)$ & Total \\
\hline$(1)$ & 44 & 18 & 28 & 8 & 6 & 8 & 1 & 5 & 118
\end{tabular}

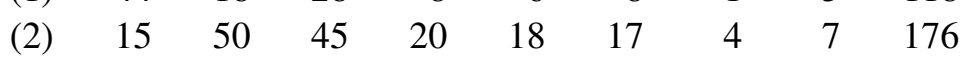

(3) $\begin{array}{lllllllll}18 & 25 & 47 & 30 & 24 & 18 & 5 & 7 & 174\end{array}$

$\begin{array}{llllllllll}\text { (4) } & 16 & 27 & 53 & 77 & 40 & 29 & 9 & 6 & 257\end{array}$

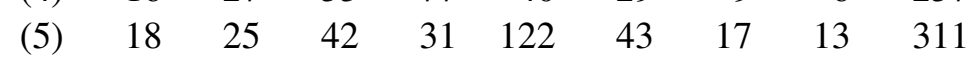

$\begin{array}{llllllllll}\text { (6) } & 12 & 15 & 21 & 15 & 36 & 33 & 3 & 8 & 143\end{array}$

$\begin{array}{lrrrrrrrrr}\text { (7) } & 3 & 5 & 8 & 7 & 26 & 21 & 9 & 3 & 82\end{array}$

\begin{tabular}{rrrrrrrrrr}
$(8)$ & 44 & 65 & 114 & 92 & 184 & 195 & 58 & 325 & 1077 \\
\hline Total & 170 & 230 & 358 & 280 & 456 & 364 & 106 & 374 & 2338 \\
\hline
\end{tabular}

Status: professional (1) managers (2) clerical (3) sales (4) skilled manual (5) semiskilled manual (6) unskilled manual (7) farmers (8)

Similarly, $\sqrt{n}\left(\hat{\Gamma}_{\mathrm{E}}-\Gamma_{\mathrm{E}}\right)$ has asymptotically a normal distribution with mean zero and variance

$$
\operatorname{var}\left(\Gamma_{\mathrm{E}}\right)=\frac{1}{\Delta^{2}} \sum_{k=1}^{r-1} \sum_{l=k+1}^{r}\left(p_{k l} \alpha_{k l}^{2}+p_{l k} \beta_{l k}^{2}\right),
$$

where

$$
\alpha_{k l}=\frac{l-k}{\log 2}\left\{\log \left(2 \Delta_{U}^{*}\right)-\Gamma_{\mathrm{E}} \log 2\right\}, \quad \beta_{l k}=\frac{l-k}{\log 2}\left\{\log \left(2 \Delta_{L}^{*}\right)-\Gamma_{\mathrm{E}} \log 2\right\} .
$$

Let $\widehat{\operatorname{var}}(\Gamma)$ denote $\operatorname{var}(\Gamma)$ with $\left\{p_{i j}\right\}$ replaced by $\left\{\hat{p}_{i j}\right\}$. Then $\widehat{\operatorname{var}}^{1 / 2}[\Gamma] / \sqrt{n}$ is the estimated approximate standard error for $\hat{\Gamma}$, giving a confidence interval for $\Gamma$.

\section{Examples}

Example 1. The data in Table 1 taken from Tominaga (1979, p.131) describe the crossclassification of father's and son's occupational status categories in Japan which were examined in 1955 and 1975. 
Table 2: Estimates of $\Gamma_{\mathrm{EMH}}$, its approximate standard error, and $95 \%$ confidence interval

\begin{tabular}{cccc}
\hline $\begin{array}{c}\text { Applied } \\
\text { data }\end{array}$ & $\begin{array}{c}\text { Estimated } \\
\text { measure }\end{array}$ & $\begin{array}{c}\text { Standard } \\
\text { error }\end{array}$ & $\begin{array}{c}\text { Confidence } \\
\text { interval }\end{array}$ \\
\hline Table 1a & 0.023 & 0.005 & $(0.013,0.034)$ \\
Table 1b & 0.055 & 0.007 & $(0.042,0.068)$ \\
\hline
\end{tabular}

Since the confidence interval for $\Gamma_{\text {EMH }}$ applied to each of Tables 1a and $1 \mathrm{~b}$ does not include zero (see Table 2), this would indicate that there is not a structure of EMH in each table. Let $G^{2}$ denote the likelihood ratio chi-squared statistic for testing goodness-of-fit of the model. The values of $G^{2}$ for the EMH model are 116.76 for Table 1a and 280.73 for Table $1 \mathrm{~b}$ with $r-2=6$ degrees of freedom (df). Therefore the EMH model fits each of these data poorly.

We compare the degree of departure from EMH between these tables using $\hat{\Gamma}_{\text {EMH }}$. We can see from $\hat{\Gamma}_{\text {EMH }}$ that (i) for Table 1a, the degree of departure from EMH is estimated to be 0.023 times the maximum degree of departure from EMH, and (ii) for Table $1 \mathrm{~b}$, it is estimated to be 0.055 times the maximum degree of departure from EMH.

When the degrees of departure from EMH in Tables $1 \mathrm{a}$ and $1 \mathrm{~b}$ are compared using the confidence interval for $\Gamma_{\mathrm{EMH}}$, it would be greater in Table $1 \mathrm{~b}$ than in Table 1a.

Table 3: Cross-classification of Merino ewes according to number of lambs born in consecutive years

\begin{tabular}{ccccc}
\hline & \multicolumn{5}{c}{ Number of Lambs 1952 } \\
Number of Lambs 1953 & 0 & 1 & 2 & Total \\
\hline 0 & 58 & 52 & 1 & 111 \\
1 & 26 & 58 & 3 & 87 \\
2 & 8 & 12 & 9 & 29 \\
\hline Total & 92 & 122 & 13 & 227 \\
\hline
\end{tabular}

Table 4: Estimates of $\Gamma_{\mathrm{EMH}}, \Gamma_{\mathrm{E}}$, and $\Gamma_{\mathrm{MH}}$, their approximate standard errors and $95 \%$ confidence intervals, applied to Table 3

\begin{tabular}{lccc}
\hline Measures & $\begin{array}{c}\text { Estimated } \\
\text { measures }\end{array}$ & $\begin{array}{c}\text { Standard } \\
\text { errors }\end{array}$ & $\begin{array}{c}\text { Confidence } \\
\text { intervals }\end{array}$ \\
\hline$\Gamma_{\mathrm{EMH}}$ & 0.102 & 0.043 & $(0.018,0.187)$ \\
$\Gamma_{\mathrm{E}}$ & 0.001 & 0.004 & $(-0.007,0.008)$ \\
$\Gamma_{\mathrm{MH}}$ & 0.103 & 0.043 & $(0.018,0.188)$ \\
\hline
\end{tabular}

Example 2. The data in Table 3 taken from Tallis (1962) describe the cross-classification of Merino ewes according to the number of lambs born in consecutive years, 1952 and 1953 (also see Bishop et al., 1975, p.288).

Since the confidence intervals for $\Gamma_{\mathrm{MH}}$ and $\Gamma_{\mathrm{EMH}}$ do not include zero (see Table 4), these would indicate that there is not each structure of $\mathrm{MH}$ and EMH in the table. How- 
ever, since the confidence interval for $\Gamma_{\mathrm{E}}$ includes zero (see Table 4), this would indicate that there is a structure of $\mathrm{E}$ in the table. Also, the degree of departure from $\mathrm{EMH}$ is larger than the degree of departure from E. Therefore we can state from Theorem 1 that the lack of structure of the MH model is caused by the lack of structure of the EMH model rather than that of the E model.

Table 5: Average doses of conjugated oestrogen used by cases and matched controls: Los Angeles endometrial cancer study

\begin{tabular}{cccccc}
\hline Average dose & \multicolumn{4}{c}{ Average dose for control (mg/day) } & \\
\cline { 2 - 5 } for case & 0 & $0.1-0.299$ & $0.3-0.625$ & $0.626+$ & Total \\
$(\mathrm{mg} /$ day $)$ & $(1)$ & $(2)$ & $(3)$ & $(4)$ & \\
\hline $0 \quad(1)$ & 6 & 2 & 3 & 1 & 12 \\
$0.1-0.299(2)$ & 9 & 4 & 2 & 1 & 16 \\
$0.3-0.625(3)$ & 9 & 2 & 3 & 1 & 15 \\
$0.626+(4)$ & 12 & 1 & 2 & 1 & 16 \\
\hline Total & 36 & 9 & 10 & 4 & 59 \\
\hline
\end{tabular}

Table 6: Estimates of $\Gamma_{\mathrm{EMH}}, \Gamma_{\mathrm{E}}$, and $\Gamma_{\mathrm{MH}}$, their approximate standard errors and $95 \%$ confidence intervals, applied to Table 5

\begin{tabular}{cccc}
\hline Measures & $\begin{array}{c}\text { Estimated } \\
\text { measures }\end{array}$ & $\begin{array}{c}\text { Standard } \\
\text { errors }\end{array}$ & $\begin{array}{c}\text { Confidence } \\
\text { intervals }\end{array}$ \\
\hline$\Gamma_{\mathrm{EMH}}$ & 0.004 & 0.006 & $(-0.009,0.016)$ \\
$\Gamma_{\mathrm{E}}$ & 0.302 & 0.125 & $(0.057,0.548)$ \\
$\Gamma_{\mathrm{MH}}$ & 0.306 & 0.124 & $(0.062,0.550)$ \\
\hline
\end{tabular}

Example 3. Table 5 taken directly from Breslow and Day (1980, p.185) is the data from the Los Angeles study of endometrial cancer. These data are obtained from the 59 matched pairs using four dose levels of conjugated oestrogen, (1) none, (2) 0.1-0.299mg, (3) $0.3-0.625 \mathrm{mg}$, and (4) $0.626+\mathrm{mg}$. Since the confidence intervals for $\Gamma_{\mathrm{MH}}$ and $\Gamma_{\mathrm{E}}$ do not include zero (see Table 6), these would indicate that there is not each structure of $\mathrm{MH}$ and $\mathrm{E}$ in the table. While, since the confidence interval for $\Gamma_{\mathrm{EMH}}$ includes zero (see Table 6), this would indicate that there is a structure of EMH in the table. In addition, the degree of departure from $\mathrm{E}$ is larger than the degree of departure from EMH. Therefore we can state from Theorem 1 that the lack of structure of the MH model is caused by the lack of structure of the E model rather than that of the EMH model. This is contrast to the case of Example 2.

\section{Concluding Remarks}

The measures $\hat{\Gamma}_{\mathrm{EMH}}$ and $\hat{\Gamma}_{\mathrm{E}}$ always range between 0 and 1 independent of the dimension $r$ and sample size $n$. So, $\hat{\Gamma}_{\mathrm{EMH}}$ and $\hat{\Gamma}_{\mathrm{E}}$ may be useful for comparing the degree of departure from $\mathrm{EMH}$ and $\mathrm{E}$, respectively, in several tables. 
Table 7: Artificial data with $\hat{G}_{1(i)} / \hat{G}_{2(i)}(i=1,2,3), \hat{\Gamma}_{\mathrm{EMH}}$, and $G^{2}$ for the EMH model

\begin{tabular}{cccc}
\multicolumn{4}{c}{ (a) $n=2611$ (sample size) } \\
\hline 511 & 216 & 9 & 45 \\
27 & 304 & 63 & 90 \\
18 & 198 & 497 & 90 \\
54 & 36 & 90 & 363 \\
\hline
\end{tabular}

\begin{tabular}{cccc}
\multicolumn{5}{c}{ (b) $n=2106$} \\
\hline 604 & 81 & 18 & 8 \\
9 & 326 & 45 & 4 \\
13 & 120 & 455 & 3 \\
6 & 4 & 2 & 408 \\
\hline
\end{tabular}

\begin{tabular}{cccc}
\multicolumn{4}{c}{ (c) $n=2419$} \\
\hline 604 & 162 & 36 & 16 \\
18 & 326 & 90 & 8 \\
26 & 240 & 455 & 6 \\
12 & 8 & 4 & 408 \\
\hline
\end{tabular}

\begin{tabular}{cccccc}
\multicolumn{4}{c}{ (d) $\left\{\hat{G}_{1(i)} / \hat{G}_{2(i)}\right\}, \hat{\Gamma}_{\mathrm{EMH}}$, and $G^{2}$ for EMH } \\
\hline & $i=1$ & 2 & 3 & $\hat{\Gamma}_{\mathrm{EMH}}$ & $G^{2}$ \\
\hline Table 7a & 2.73 & 0.68 & 1.25 & 0.054 & 154.17 \\
Table 7b & 3.82 & 0.52 & 1.25 & 0.134 & 99.75 \\
Table 7c & 3.82 & 0.52 & 1.25 & 0.134 & 199.50
\end{tabular}

Note: $\hat{G}_{1(i)} / \hat{G}_{2(i)}$ indicates $G_{1(i)} / G_{2(i)}$ with $\left\{p_{s t}\right\}$ replaced by $\left\{\hat{p}_{s t}=n_{s t} / n\right\}$.

Consider the artificial data in Table 7. From the values of $G^{2}$ (with 2 df) for the EMH model (see Table 7d), we see that the EMH model fits the data in Table 7a worse than the data in Table $7 \mathrm{~b}$. In contrast, the value of $\hat{\Gamma}_{\mathrm{EMH}}$ is less for Table $7 \mathrm{a}$ than for Table $7 \mathrm{~b}$ (see Table $7 \mathrm{~d}$ ). In terms of $\left\{\hat{G}_{1(i)} / \hat{G}_{2(i)}\right\}, i=1,2,3$ (see Table 7d), it seems natural to conclude that the degree of departure from EMH is less for Table 7a than for Table $7 \mathrm{~b}$. Therefore $\hat{\Gamma}_{\mathrm{EMH}}$ may be preferable to $G^{2}$ for comparing the degree of departure from EMH in several tables. (By the similar reason, $\hat{\Gamma}_{\mathrm{EMH}}$ may also be preferable to the $P$-values for comparing them.)

It may seem, to many readers, that $G^{2} / n$ is also a reasonable measure for representing the degree of departure from EMH. However, it does not seem to us that $G^{2} / n$ is a reasonable measure. For example, consider the artificial data in Tables $7 \mathrm{~b}$ and $7 \mathrm{c}$. The values of $G^{2} / n$ are 0.05 for Table $7 \mathrm{~b}$, and 0.08 for Table $7 \mathrm{c}$. Therefore the value of $G^{2} / n$ is less for Table $7 \mathrm{~b}$ than for Table $7 \mathrm{c}$. However, the value of $\hat{\Gamma}_{\mathrm{EMH}}$ for Table $7 \mathrm{~b}$ is theoretically identical to that for Table $7 \mathrm{c}$ because $\left\{\hat{G}_{1(i)} / \hat{G}_{2(i)}\right\}, i=1,2,3$, for Table $7 \mathrm{~b}$ is identical to those for Table 7c (see Table 7d). It seems natural to conclude that the degree of departure from EMH for Table $7 \mathrm{~b}$ is equal to that for Table $7 \mathrm{c}$. Therefore $\hat{\Gamma}_{\text {EMH }}$ may also be preferable to $G^{2} / n$ for comparing the degree of departure from EMH in several tables.

The $\hat{\Gamma}_{\text {EMH }}$ would be useful when we want to see what degree the departure from EMH is toward the maximum departure from EMH. Note that we cannot use the $G^{2}$ and $\hat{\Gamma}_{\mathrm{MH}}$ when we want to see it.

We observe that (i) the measure $\Gamma_{\mathrm{EMH}}$ should be applied to contingency tables with ordered categories, (ii) the asymptotic normal distribution of $\sqrt{n}\left(\hat{\Gamma}_{\mathrm{EMH}}-\Gamma_{\mathrm{EMH}}\right)$ may be not applicable when $\Gamma_{\mathrm{EMH}}=0$ and $\Gamma_{\mathrm{EMH}}=1$ because then $\operatorname{var}\left[\Gamma_{\mathrm{EMH}}\right]=0$, and (iii) $\hat{\Gamma}_{\text {EMH }}$ cannot be used for testing goodness-of-fit of EMH (although $G^{2}$ can be used for it). 
Table 8: Artificial data

\begin{tabular}{cccc}
\multicolumn{4}{c}{$n=1169$ (sample size) } \\
\hline 100 & 54 & 50 & 46 \\
8 & 120 & 250 & 104 \\
8 & 20 & 150 & 50 \\
4 & 13 & 12 & 180 \\
\hline
\end{tabular}

\section{Discussion}

Consider the artificial data in Table 8. The values of $G^{2}$ for the MH and EMH models are 389.17 with $3 \mathrm{df}$ and 4.91 with $2 \mathrm{df}$, respectively. Therefore, these would indicate that for these data, there is not the structure of $\mathrm{MH}$, however, there is the structure of EMH. Then the estimated value of measure $\Gamma_{\mathrm{MH}}$ is 0.517 and the estimated value of measure $\Gamma_{\mathrm{EMH}}$ is 0.002. Thus, when we want to see the degree of departure from $\mathrm{EMH}$, the measure $\Gamma_{\mathrm{EMH}}$ would be useful although the measure $\Gamma_{\mathrm{MH}}$ is not useful.

We emphasize that the measure $\Gamma_{\mathrm{EMH}}$ proposed in this paper is entirely different from the measures which represent the degree of departure from $\mathrm{MH}$ as the measure $\Gamma_{\mathrm{MH}}$ although the form may be similar.

Finally we note that Tomizawa et al. (2003) also gave the power-divergence type measure to represent the degree of departure from $\mathrm{MH}$; however, using the power-divergence, we cannot obtain the similar result to Theorem 1.

\section{Acknowledgments}

The authors would like to thank a referee for many helpful comments.

\section{Appendix}

The KL information type measure $\Gamma_{\mathrm{MH}}$, which represents the degree of departure from MH for the data on an ordinal scale, proposed in Tomizawa et al. (2003), is given as follows: assuming that $\left\{G_{1(i)}+G_{2(i)}>0\right\}$,

$$
\Gamma_{\mathrm{MH}}=\frac{1}{\log 2} I\left(\left\{G_{1(i)}^{*}, G_{2(i)}^{*}\right\} ;\left\{Q_{i}^{*}, Q_{i}^{*}\right\}\right),
$$

where

$$
\begin{gathered}
I(\cdot ; \cdot)=\sum_{i=1}^{r-1}\left\{G_{1(i)}^{*} \log \left(\frac{G_{1(i)}^{*}}{Q_{i}^{*}}\right)+G_{2(i)}^{*} \log \left(\frac{G_{2(i)}^{*}}{Q_{i}^{*}}\right)\right\}, \\
Q_{i}^{*}=\frac{1}{2}\left(G_{1(i)}^{*}+G_{2(i)}^{*}\right) .
\end{gathered}
$$

Note that $0 \leq \Gamma_{\mathrm{MH}} \leq 1$, and $\Gamma_{\mathrm{MH}}=0$ if and only if the $\mathrm{MH}$ model holds. 


\section{References}

Agresti, A. (1990). Categorical Data Analysis. New York: John Wiley.

Bishop, Y. M. M., Fienberg, S. E., and Holland, P. W. (1975). Discrete Multivariate Analysis: Theory and Practice. Cambridge, Massachusetts: The MIT Press.

Breslow, N. E., and Day, N. E. (1980). Statistical Methods in Cancer Research, Vol. IThe Analysis of Case-Control Studies. Lyon, France: International Agency for Research on Cancer.

Stuart, A. (1955). A test for homogeneity of the marginal distributions in a two-way classification. Biometrika, 42, 412-416.

Tallis, G. M. (1962). The maximum likelihood estimation of correlation from contingency tables. Biometrics, 18, 342-353.

Tominaga, K. (1979). Nippon no Kaisou Kouzou (Japanese Hierarchical Structure). Tokyo: University of Tokyo Press. (in Japanese)

Tomizawa, S. (1991). Decomposing the marginal homogeneity model into two models for square contingency tables with ordered categories. Calcutta Statistical Association Bulletin, 41, 201-207.

Tomizawa, S. (1993). Diagonals-parameter symmetry model for cumulative probabilities in square contingency tables with ordered categories. Biometrics, 49, 883-887.

Tomizawa, S. (1995). Measures of departure from marginal homogeneity for contingency tables with nominal categories. Journal of the Royal Statistical Society, Series D, The Statistician, 44, 425-439.

Tomizawa, S., and Makii, T. (2001). Generalized measures of departure from marginal homogeneity for contingency tables with nominal categories. Journal of Statistical Research, 35, 1-24.

Tomizawa, S., Miyamoto, N., and Ashihara, N. (2003). Measure of departure from marginal homogeneity for square contingency tables having ordered categories. Behaviormetrika, 30, 173-193.

Authors' address:

Kouji Yamamoto and Sadao Tomizawa

Department of Information Sciences

Faculty of Science and Technology

Tokyo University of Science

Noda City, Chiba, 278-8510

Japan

E-mail: tomizawa@is.noda.tus.ac.jp 